\title{
¿Qué son y dónde se sitúan los Estudios Feministas de Seguridad?
}

\section{What, and Where, is Feminist Security Studies?}

\author{
LAURA SJOBERG*
}

\section{RESUMEN}

Esta obra echa la mirada hacia el pasado y hacia el futuro en relación con lo que fue, es y podría ser el trabajo feminista en seguridad, mediante la combinación de un enfoque de sociología histórica con una visión prospectiva del futuro de este campo de estudio. El texto comienza con una reflexión, como cimiento de la discusión, sobre los estudios feministas de seguridad (EFS) antes de la existencia misma de la disciplina.A continuación, discute diferentes ideas sobre lo que los EFS son. Posteriormente, presta atención a las corrientes divergentes dentro los EFS, así como a las omisiones y críticas. Más que buscar reconciliar estas distintas consideraciones, se pregunta por los aspectos que puede tomarse de las mismas para abordar futuros potenciales de los EFS, y su contribución a los feminismos y/o a los estudios de seguridad.

\section{Abstract}

This piece looks to backwards and forwards to what feminist work in security was, is, and could be, pairing a historical sociology with a forward-looking view of the future(s) of the field. It begins with thinking about feminist studies of security before FSS as a foundation for the discussion, then traces different claims to core identities of FSS. It then looks at divergent strands of FSS, as well as omissions and critiques. Rather than looking to reconcile those different accounts, it asks what can be taken from them to engage potential futures for FSS, and its contribution to feminisms and/or studies of security.

\section{ntroducción}

[Los Estudios Feministas de Seguridad pretenden] desafiar la supuesta irrelevancia del género... [para] argumentar que el género no es una subsección de los Estudios de Seguridad que debe ser objeto de compartimentalización o brevemente considerada como una cuestión secundaria. Más bien, las feministas argumentan que el género es conceptualmente, empíricamente, y normativamente esencial para el estudio de la seguridad internacional. Como tal, una

Este texto fue publicado originalmente en 2016 en la revista Journal of Regional Security, vol. II, nro. 2, pp. $|43-16|$ con el título "What, and Where, is Feminist Security Studies?" (DOI: 10.1 I643/issn.2217-995XI62SPS66). La autora del texto agradece a la revista Journal of Regional Security por el ímpetu puesto desde un primer momento para que pudiera escribir este texto y para que se lleve a cabo su traducción a lengua castellana, así como a la Academia Británica por su apoyo durante las revisions del texto. Esta revisión acerca del estado de los Estudios Feministas de Seguridad se limita a la literatura de habla inglesa sobre éstos y, por lo tanto, ha omitido potenciales diálogos con investigadores e investigadoras y desarrollos por parte de obras no pertenecientes a la literatura de habla inglesa sobre la cuestión.

DOI:

https://doi.org/|0.15366/relacionesinternacionales202I.48.00I

Formato de citación recomendado:

SJOBERG, Laura (202I). “Qué son y dónde se sitúan los Estudios Feministas de Seguridad?”, Relaciones Internacionales, $n^{\circ} 48$, pp. I5-30.

\section{* Laura} SJOBERG,

Es Global Professor de Política y Relaciones Internacionales de la Academia Británica, en la Universidad Royal Holloway de Londres, y profesora de Ciencias Políticas en la Universidad de Florida. Su investigación aborda temas de género y seguridad, con especial atención a la violencia política perpetrada por mujeres, la teorización feminista de la guerra, la sexualidad en la política global y la metodología política. Enseña, asesora y da conferencias sobre género en la política global y sobre seguridad internacional. Su trabajo ha sido publicado en más de cincuenta libros y revistas de Ciencias Políticas, Derecho, Estudios de Género, Relaciones Internacionales y Geografía.

Traducción: Victoria SILVA SÁNCHEZ 
producción académica precisa, rigurosa, y ética no puede ser desarrollada sin tomar en consideración la presencia de las mujeres o la incorporación del enfoque de género a la política internacional. (Sjoberg, 2009, p. 186).

Cuando comencé a pensar y escribir sobre la seguridad internacional desde una perspectiva feminista, el término "estudios feministas de seguridad", no se usaba, hasta donde mi saber alcanza, como un término técnico, aunque aparecía de forma excepcional en algunos textos. Esto no significa que las académicas feministas no estudiasen la seguridad internacional - de hecho, lo hacían, quizás, de una manera más extensa de lo que muchas lo hacemos hoy en día- Lo que significa es que el trabajo feminista en seguridad antecede, continúa a las afueras, y, con suerte, permanecerá, más allá del uso del término Estudios Feministas de Seguridad (EFS de aquí en adelante). Esta obra echa la mirada hacia el pasado y hacia el futuro en relación con lo que fue, es y podría ser el trabajo feminista en seguridad, mediante la combinación de un enfoque de sociología histórica con una visión prospectiva del futuro de este campo de estudio. El texto comienza con una reflexión, como cimiento de la discusión, sobre los estudios feministas de seguridad (EFS) antes de la existencia misma de la disciplina. A continuación, discute diferentes ideas sobre lo que los EFS son. Posteriormente, presta atención a las corrientes divergentes dentro los EFS, así como a las omisiones y críticas. Más que buscar reconciliar estas distintas consideraciones, se pregunta por los aspectos que puede tomarse de las mismas para abordar futuros potenciales de los EFS, y su contribución a los feminismos y/o a los estudios de seguridad.

\section{Género y seguridad 'fuera' de las Relaciones Internacionales}

El trabajo feminista llegó relativamente tarde a las Relaciones Internacionales (RRII) y la ciencia política, en comparación con muchas otras ciencias sociales y humanidades, así como con el 'mundo real' de la política global. No se trata, entonces, simplemente de que el trabajo feminista en seguridad en las RRII precede a los EFS en las RRII, sino de que el trabajo feminista en seguridad antecede al trabajo feminista en las RRII.

De hecho, considero que un argumento justo a este respecto apunta a que la academia feminista, y el activismo feminista, tratan fundamentalmente sobre la seguridad. Las definiciones estándar de los diccionarios de seguridad sugieren que se trata del grado de resistencia contra, o de protección frente al daño. Los primeros feminismos en el siglo XIX y comienzos del siglo XX que buscaban cambiar las desigualdades legales de la mujer, particularmente su (in)capacidad de votar en las democracias electorales y su (falta de) acceso a derechos de propiedad, educativos y de empleo, trataban, fundamentalmente, de oponer resistencia contra la distribución del daño (denegación de oportunidades) de acuerdo con criterios de sexo. Lo que comúnmente se denomina 'segunda ola' del feminismo en Estados Unidos y Europa Occidental —un movimiento que mantuvo el compromiso con la igualdad legal, pero amplió los feminismos para cuestionar los roles tradicionales de la mujer en la vida política y social, y criticar los roles de género heredados - perseguía la reparación del daño causado a las mujeres como resultado de, y en relación con, las normas de género que estructuraban sus vidas. Lo que comúnmente se conoce como 'tercera ola' del feminismo incluye esos intereses, y presta mayor atención a los daños interseccionales — la subordinación de género interrelacionada con la discriminación basada en 
la clase, raza, sexualidad y nacionalidad - de nuevo interesándose por la protección respecto de, $y$ en poner fin al daño.

Existen razones tanto estratégicas como intelectuales para no tratar el feminismo en sí mismo como una teoría y práctica de seguridad —en particular, los bien documentados potenciales efectos nocivos de la securitización (p. ej., Buzan, Waever y de Wilde, 1998)—. La securitización — designar algo como seguridad - puede tener un efecto de militarización - cambiar las categorías tradicionales de seguridad sin cambiar las herramientas (militares) tradicionales utilizadas para abordar lo que entendemos como seguridad-. Asociar los feminismos como movimientos interesados en proveer y analizar el potencial de proporcionar seguridad tiene como impacto, correcto o no, asociar esos feminismos con militarismos, dada la asociación permanente entre seguridad y militarismo.

Dicho esto, el trabajo feminista fuera de lo que es tradicionalmente considerado como RRII ha mostrado una profunda preocupación por cuestiones de género y seguridad. Desde la crítica mordaz del derecho internacional de los derechos humanos de Catherine MacKinnon (2006) —Are Women Human? - a las demandas de justicia para las mujeres Negras de Patricia Hill Collins (1998) —FightingWords - una gran variedad de feministas han llamado la atención hacia las varias formas en las que la subordinación de género amenaza la seguridad. Estos reconocimientos tampoco son nuevos — pueden ser identificados en la obra de Jane Addams (1910) Twenty Years at Hull House (que vincula pobreza, violencia doméstica y subordinación de género); “Ain't I a Woman?" de Sojourner Truth (185I) (que argumenta que el feminismo no debería centrarse principalmente en los problemas enfrentados por las mujeres blancas); y la obra de Qiu Jin (1906) Respectful Proclamation to China's 200 Million Women Comrades (que condena la violencia de género y las prácticas de vendaje de pies) - Las formas en que el género (especialmente cómo éste intersecta con la raza y la clase) distribuye las amenazas a la seguridad ha representado la materia subyacente de una cantidad significativa de textos académicos feministas y acciones de activismo desde que los feminismos existen.

\section{Los Estudios Feministas de Seguridad antes de los EFS}

Estos temas son incorporados cuando comienza a surgir la producción académica feminista en el estudio de la política global. El libro de Ann Tickner (1992), ampliamente reconocido como el primer libro de RRII escrito por un único autor explícitamente feminista, tenía como título Gender in International Relations: Feminist Perspectives on Achieving Global Security. En otras palabras, la seguridad fue la base de la aplicación por parte de Tickner de la teoría feminista a las relaciones internacionales. El libro de Tickner reunió una combinación de su formación en estudios de desarrollo, sus lecturas de filosofía de la ciencia feminista (p. ej., Keller 1985; Harding 1986), teorización de la paz feminista (p. ej., Reardon 1985; Elshtain 1985) y teorización de las RRII sobre guerra y paz (p. ej., Morgenthau 1948; Waltz 1959), economía política (p. ej., Keohane 1984), y política medioambiental (p. ej., Haas 1990). Gendered States, editado por Spike Peterson (1992) representaba, en gran parte, un estudio de las formas en las que la influencia del género en la política global constituye las maneras en las que los estados proveen, o afirman proveer, de seguridad a sus ciudadanos. "Under Western Eyes" de Chandra Mohanty (1984) identifica las 
numerosas maneras en las que no sólo la política malestream, sino también los discursos feministas occidentales colonizan y violentan el constructo de "Mujer del Tercer Mundo" y a las mujeres para cuya descripción esa categoría es desplegada. Muchas obras tempranas de las RRII feministas (p. ej., Whitworth 1997; Sylvester 1994; Pettman 1996) abarcan una cantidad significativa de análisis del tema en cuestión que podrían ser tradicionalmente entendidos por parte de las RRII ortodoxas como 'seguridad', sin por ello llevar la etiqueta "Estudios Feministas de Seguridad".

Dos características de este trabajo me resultan de interés con respecto a este texto. La primera característica del trabajo es la medida en la que combina cuestiones de seguridad con la economía política, los derechos humanos, y las cuestiones medioambientales. Por ejemplo,Tickner (1992) identificó el conflicto, la economía y el medio ambiente en conjunto como tres aspectos esenciales para "alcanzar la seguridad global”. Bananas, Beaches, and Bases de Enloe (I989) dedica un esfuerzo considerable a discutir lo que ahora denominamos la economía política de la seguridad (las formas en las que la militarización moldea las relaciones económicas) y la securitización de la economía política (las formas en las que los patrones económicos son entendidos como parte de la seguridad nacional y/o personal). Nira Yuval-Davis (1997) habló sobre las maneras en las que la seguridad estatal dependía de las mujeres como reproductores biológicos y culturales de la identidad estatal/nacional, un discurso desarrollado en investigaciones subsecuentes sobre la discusión en torno al trabajo productivo y reproductivo feminizado (p. ej., Peterson 20l0). La discusión feminista en torno a que lo personal es político (en palabras de Enloe [2004], “lo personal es internacional y lo internacional es personal) motivó la teorización de intersecciones que previamente habían sido invisibles o poco estudiadas en la teoría de RRII — vínculos entre el centro de la política global y los márgenes (p. ej., Moon 1997), vínculos entre la economía política, la seguridad personal, y la seguridad internacional (p. ej., Enloe 20l0), y vínculos entre el género, la raza, y la clase en la seguridad (p. ej.,Yuval-Davis 1997)—. Todas estas contribuciones a los estudios feministas de seguridad ( $y$ a los feminismos y los estudios de seguridad) surgieron antes de que la nomenclatura 'Estudios Feministas de Seguridad' fuese ampliamente utilizada.

La segunda característica de esta obra es que, cuando fue reconocida por la más amplia disciplina de Relaciones Internacionales, fue raramente reconocida como una contribución al estudio de la seguridad. Existen por supuesto excepciones — por ejemplo, la tipología de Steve Smith (2005) de los componentes de los Estudios Críticos de Seguridad menciona los Estudios Feministas de Seguridad como un tipo-. Aun así, la revista Security Studies, por ejemplo, no publicó las palabras mujer o género hasta bien entrado el siglo XXI, e International Security lo hizo de forma irregular, y generalmente sin hacer referencia a la producción feminista de RRII que prestaba atención a la seguridad. El trabajo de RRII que reconocía el feminismo con frecuencia lo veía dentro de la esfera de las políticas de identidad, los constructivismos, o los derechos humanos. Las investigaciones en Estudios de Seguridad generalmente funcionaban como si el trabajo feminista en el campo de estudio no existiese, y el reconocimiento del trabajo feminista en RRII, cuando tenía lugar, era normalmente tratado como un enfoque teórico paradigmático a la teorización en materia de política global — realismo, liberalismo, constructivismo, feminismos, etc- Como resultado, las contribuciones feministas al pensamiento sobre la seguridad en la política global a menudo, voluntaria o involuntariamente, dialogaban entre si -especialmente en lo que respecta al significado y las dinámicas de la seguridad-. 


\section{La aparición de (el término) Estudios Feministas de Seguridad}

Fue esta segunda característica — la falta de reconocimiento del trabajo feminista en seguridad como trabajo en seguridad - la que motivó que empezara a utilizar el término 'Estudios Feministas de Seguridad' como un término técnico. Tras intentar y fracasar, en un encargo de consultoría, a la hora de delinear las contribuciones feministas al campo de los Estudios de Seguridad (llamada así por mi entonces empleador, no por mi), comencé a preguntarme por qué se había producido un diálogo tan escaso entre estos programas de investigación -más particularmente, por qué los 'Estudios de Seguridad' incluían tan poco feminismo-. Me di cuenta de que, a pesar de que las feministas habían realizado importantes investigaciones sobre el género, la guerra y el conflicto durante décadas, su trabajo no había sido incluido en las listas de lectura o los coloquios de investigadores de los Estudios de Seguridad. Como he explicado anteriormente, a la sazón "utilicé el término 'Estudios Feministas de Seguridad' con la intención explícita de convencer a las personas de los Estudios de Seguridad de que el trabajo feminista era relevante para sus investigaciones" (Sjoberg, 20I5). Mi utilización del término miraba hacia el exterior: existía un campo de Estudios de Seguridad, y consideraba que el trabajo basado en el género debía ser una parte importante del mismo. En consecuencia, en 2006 propuse un monográfico a la revista Security Studies (Sjoberg, 2009) y un seminario relacionado con éste en la Annual Meeting of the International Studies Association de 2007.

Mis reflexiones en aquel momento buscaban la manera de hacer visibles las formas en las que el trabajo feminista no solo criticaba, sino que también reconstruía, reconstituía, y transformaba el objeto de estudio de los Estudios de Seguridad, así como los métodos de los que el campo podía valerse para llevar a cabo la tarea de estudiar la seguridad en la política global. Siguiendo la crítica de Ann Tickner (1988; 1997) de que la esfera de "poder y conflicto" en la política global es "un mundo de hombres" en el que el análisis de género frecuentemente se topa con "silencios incómodos y mala comunicación", tenía como objetivo — junto con Spike Peterson (1992, p. 197) — abordar de frente "las perspectivas androcéntricas que hacen invisibles a las mujeres y a las relaciones de género". Mi uso del término tenía como "objetivo mejorar la calidad y cantidad de las conversaciones entre los Estudios de Seguridad feministas y los Estudios de Seguridad en general, para demostrar la importancia del análisis de género en el estudio de la seguridad internacional, y así expandir el programa de investigación feminista en los Estudios de Seguridad” (Sjoberg, 2009, p. 188).

Otros usos del término durante aquel periodo, e incluso con anterioridad, mostraban un compromiso menor con el gigante disciplinario de los Estudios de Seguridad, y estaban más interesados en lo que los feminismos pensaban sobre y/o aportaban al pensamiento sobre la seguridad en la esfera política global. Por ejemplo, Jasmina Husanovic y Patricia Owens (2000, p. 425 , p. 434,) sugieren que unos estudios feministas/de seguridad representarían una exploración y/o intervención interesante:"en este punto, lo que puedan parecer unos 'estudios feministas de seguridad' es incierto... quizás lo primero es proporcionar un control negativo y limitante de los hechos erróneos". Esto enmarcó los 'estudios feministas de seguridad' menos como un término técnico y más como una orientación ética hacia las injusticias (de género) en el mundo. Lene Hansen (2000, p. 70n3) citando a Tickner (1997, p. 628) también describe los "estudios feministas de seguridad" como una orientación interesada en la seguridad con un enfoque "desde la base". 
Mi opinión es que tanto un uso enfocado hacia adentro como hacia afuera del término y/o de la agrupación Estudios Feministas de Seguridad puede tomar en cuenta estas orientaciones - y así lo ha hecho-.

Por ejemplo, mi descripción inicial de los EFS los describía como el reconocimiento de una relación entre el conocedor y lo conocido, y añadía que compartían cuatro ideas comunes sobre qué es la seguridad internacional y cómo funciona dentro de la investigación feminista sobre seguridad (Sjoberg, 2009, p. 195). Estas cuatro ideas comunes eran que la seguridad debe ser entendida de forma amplia, que los valores de género son constitutivos de las normas en la esfera de seguridad, que el género juega un papel amplio y diverso en la constitución de la teoría y práctica de la seguridad, y que omitir el análisis de género o las prácticas conscientes del género están determinadas en función del género en lugar de ser neutrales respecto al género (Sjoberg, 2009, pp. 200-202). Mi interés se centraba en recopilar los conocimientos del trabajo feminista en seguridad que era ignorado por los Estudios de Seguridad en aquel momento, y empujarlos a dialogar con los Estudios de Seguridad, y para ello dar un nombre al campo en el que las feministas trabajaban en seguridad como parte de los Estudios de Seguridad.

Otros, que utilizaron el término de forma temprana en el proceso de popularización como un término técnico, tenían diferentes orientaciones respecto a lo que querían lograr con su uso. De forma igual a cómo yo lo utilizaba, aspiraban a crear espacios y articular conversaciones a través de la obra que se había constituido ya como un campo como consecuencia del uso del término. Dicho esto, muchos aspiraban a generar diferentes conversaciones. Annick Wibben (2010), por ejemplo, dirigió su discusión sobre los EFS hacia "un enfoque narrativo", en el que las narrativas sobre la seguridad en función del género son "una forma primaria de dar sentido al mundo que nos rodea". Los EFS de Wibben están orientados, en mayor medida, hacia las académicas feministas y el trabajo crítico en los Estudios de Seguridad; su uso del término mira más hacia adentro que el mío. Laura Shepherd (2009, p. 209) posiciona los EFS fuera de, pero vinculados con, las RRII/Estudios de Seguridad, y argumenta que "ofrece un correctivo importante a las asunciones fundacionales de las RRII, las cuales perpetúan los mismos casos de violencia que buscan reparar". Esta orientación y despliegue tienen un propósito y dirección diferentes que la utilización que yo hago del término, pero aún presentan una cantidad significativa de puntos en común con lo que yo creo que los EFS son, contienen, y se esfuerzan por hacer en términos de investigación. Shepherd (2009, p. 209) pasa a describir lo que ella ve como "los conocimientos básicos de los estudios feministas de seguridad" - "la centralidad del sujeto humano, la importancia de las configuraciones particulares de masculinidad y feminidad, y el marco conceptual en función del género que sustenta la disciplina de las RRII"- - La razón de ser de los EFS, señala Shepherd (2009, p. 209), es “imaginar una política bastante diferente de lo global”.

Independientemente de estas diferentes orientaciones en torno a los primeros usos del término, y de la autocomprensión del campo, los Estudios Feministas de Seguridad, tanto la orientación general a la investigación como el uso específico del término, se han popularizado. En términos de los RRII/Estudios de Seguridad disciplinarios tradicionales, en 2009 concluí que "menos de cuarenta de más de cinco mil artículos publicados en las cinco principales revistas de seguridad en los últimos veinte años abordaban de forma explícita el género como un tema sustantivo principal", incluyendo en mi análisis a International Security, Security Studies, Issues in 
International Security, International Security Review, y Security Dialogue entre 1986 y 2006 (Sjoberg, 2009, p. 187). Más de la mitad de esos artículos fueron publicados en Security Dialogue durante el siglo XXI, y, hasta aquel momento, Security Studies nunca había publicado, de acuerdo con mis resultados, la palabra 'mujer', ni siquiera incidentalmente. Desde entonces, muchas revistas que los académicos de los Estudios de Seguridad ven como sus plataformas lógicas de publicación, incluyendo las cinco revistas mencionadas anteriormente, pero también Journal of Conflict Resolution, International Interactions, American Political Science Review, y una gran variedad de revistas generalistas, han publicado trabajos que abordan explícitamente el género y la seguridad, algunos de los cuales se autoidentifican con la etiqueta EFS.

El uso de la etiqueta EFS también ha proliferado.Además de un subcampo y/o una orientación intelectual, los EFS se han convertido en un grupo de Facebook con miles de miembros, un hashtag de Twitter, y una importante oleada de paneles en las reuniones anuales de la International Studies Association cada año desde 2008. Antes de 1990, no he identificado usos del término "estudios feministas de seguridad" en medios impresos. Entre 1990 y 2006, he identificado menos de veinte trabajos académicos que utilizan esta expresión. Entre 2007 y 2020, he identificado I 3 I 0 usos publicados de esa expresión. Incrementos similares pueden ser identificado en los planes de estudios y programas de conferencias. Una disciplina EFS - quizás tanto como un subcampo de las RRII feministas como un subcampo de los Estudios de Seguridad — ha visto la luz durante las dos últimas décadas, y ha crecido rápidamente. Aun así, dónde está y dónde estará el campo está lejos de estar decidido, y qué debería ser se abre también a un importante debate.

\section{Lidiando con los Estudios Feministas de Seguridad}

A medida que los EFS se han vuelto más populares y han sido incluidas en mayor medida en las RRII (feministas), han surgido una serie de debates y cuestiones acerca de qué son, qué serán, y qué deberían ser los EFS —-debates sobre qué significa o debería significar realizar análisis feministas en/de la seguridad-. Esta sección delinea de forma breve tres grandes debates. No me sumerjo en esta tarea para sugerir que sólo hay tres grandes debates, o que las voces aquí citadas son representativas tanto del campo como de los desacuerdos dentro del mismo. Al contrario, pretendo destacar algunas áreas de desacuerdo que considero constitutivas del campo,y esbozar mi lectura de cada uno de estos desacuerdos para perfilar, en última instancia, mi opinión sobre cómo el campo podría o debería avanzar. En primer lugar, examino las inquietudes sobre si los EFS están y/o deberían estar orientados hacia las RRII ortodoxas. En segundo lugar, analizo las inquietudes sobre cómo de representativos son los EFS, tanto en términos de académicas que participan en dicho empeño, como en términos de la investigación producida por dichas académicas. En tercer lugar, repaso lo que se gana y se pierde en vista de lo que ha evolucionado como una bifurcación de los EFS y la Economía Política Feminista (EPF).

\section{Estudios Feministas de Seguridad y Estudios de Seguridad/RRII}

Como mencioné previamente, mi interés inicial en los EFS reposaba en llegar desde el trabajo feminista en RRII interesado en la seguridad a los Estudios de Seguridad/RRII disciplinarios. Este 
movimiento en sí mismo, incluso si no hubiera tenido efectos imprevistos, no está exento de contestación política en el campo, tanto antes como después de la génesis del subcampo de los EFS. De hecho, incluso algunos trabajos tempranos en las RRII feministas sugerían que había un "peligro" en la "aceptación acrítica" por parte de las feministas de los métodos, delimitaciones, ideas y temas en cuestión de las RRII tradicionalmente entendidas (Brown, 1988). Sarah Brown (1988) argumentó que los intentos por parte de las RRII feministas de comprometerse con unas RRII ortodoxas masculinistas podían representar el riesgo de perder el valor añadido crítico de las RRII feministas, especialmente cuando se convirtió en una preocupación política para las personas en los márgenes de la política global. Aunque no creo que sea justo considerar tanto el mío como otros trabajos orientados a la corriente principal como "aceptaciones acríticas", el argumento de Brown sobre los riesgos intelectuales y políticos del compromiso son dignos de ser tenidos en cuenta, $y$ han sido repetidos más recientemente de forma matizada.

Por ejemplo,AnnickWibben (2010) argumentaba que los EFS no deberían estar orientados a la corriente principal, exponiendo lo que caracterizaba como "un alegato para reconocer los peligros que implica establecer los EFS [estudios feministas de seguridad] como un subcampo de los estudios de seguridad cuando los objetivos y el alcance feministas continúan explotando sus confines en todo momento". En otras palabras, Wibben argumentaba que los EFS no pueden ser contenidos por, o en, las RRII ortodoxas ya que, en el mejor de los casos, los EFS rompen las reglas y límites de sus modos de investigación positivistas, estatistas y masculinistas. Por ello, Wibben (2010) señala que las feministas deben "avanzar más allá de una expansión y profundización de los estudios de seguridad hacia una apertura" que implicaría "una crítica continúa, radical y deliberada", "fiel a los compromisos políticos y metodológicos feministas." La parte más fundamental de esta necesidad de mantener la diferenciación, en opinión de Wibben (2010), es la cuestión de avanzar los parámetros de investigación. Por ello, Wibben (20l0) insta a que "las académicas feministas de seguridad no puedan permitir que los tradicionalistas posean la verdad indiscutible sobre a qué se refiere la seguridad, especialmente en vista de que la seguridad es tan poderosa cuando es evocada".

La sugerencia de Wibben es que existe una desconexión fundamental entre éste y otros trabajos más radicales. Considero que la discusión de Carol Cohn (20I I) en torno a esta cuestión como un problema de orientación representa un marco útil para pensar en esta contestación. En su reflexión de los Estudios Feministas de Seguridad como un término técnico, Cohn (201I, p. 582) argumenta que "existe — potencialmente al menos - una ambigüedad inherente en la propia etiqueta, relacionada con qué dos de las tres palabras uno ve como más estrechamente relacionadas." En otras palabras, a Cohn le interesa saber si los EFS son estudios de 'seguridad feminista' o 'estudios de seguridad' feministas. La primera yuxtaposición etimológica implica que los EFS son el estudio de una noción feminista de la seguridad, mientras que la segunda implica que los EFS son perspectivas feministas de una noción predefinida de 'estudios de seguridad.'

Quizás, en este esquema, ni el enfoque de Wibben ni el mío encajan perfectamente en una u otra categoría, pero el deWibben puede corresponderse más estrechamente con el enfoque de estudios de 'seguridad feminista', mientras que el mío aparece más vinculado con el enfoque de 'estudios de seguridad' feministas. Cohn considera que existen beneficios potenciales en los dos enfoques. Abordando los EFS en los que 'feminista' modifica 'estudios de seguridad', Cohn (20I I, 
p. 582) considera:

¿Implica el término Estudios Feministas de Seguridad que es necesario repensar la dinámica causal central que subyace a las principales preocupaciones dentro de los estudios de seguridad (convencionales)? Y, ¿acaso se produciría una transformación fructífera por el hecho de incluir el análisis feminista en el campo? De nuevo, en este caso, se aceptarían los límites de la autodefinición convencional del campo, pero la sugerencia es que (al menos algunos de) los supuestos centrales y análisis han sido incorrectos. Si ese es el caso, cuando "Feminista" modifica "Estudios de seguridad", entonces ¿no aspira a ser un subcampo sino una escuela? ¿O incluso un cambio de paradigma?

Cohn (20II) también explora la utilidad de reflexionar sobre la "seguridad feminista" como sujeto de los EFS. La autora sugiere que, en esta construcción, "'Seguridad Feminista' se convierte en el sujeto sobre el que los estudios se crean" (Cohn 20II, p. 582). Aunque, en opinión de Cohn (20II, p. 582), 'seguridad feminista' "es un término sin un significado obvio", tiene un potencial crítico como concepto a implementar. Entre otras sugerencias, Cohn (20l I, p. 582) propone que "el término seguridad feminista [puede] hace[r] referencia al reconocimiento feminista de que la seguridad no puede provenir de una independencia armada fantaseada, aislada, completamente autónoma y autosuficiente, sino que siempre, inevitablemente, es relacional, basada en la interdependencia." En otras palabras, este tipo de EFS pueden reorientar los estudios de seguridad a reflexionar sobre la propia constitución del concepto de seguridad (y por lo tanto del campo que estudia la seguridad) de modo diferente.

A fin de cuentas, Cohn (20ll, p. 583) evade elegir entre estas dos lecturas de las combinaciones de palabras en FSS. En su lugar, sugiere que "la pregunta no es tanto " Qué son los Estudios Feministas de Seguridad como campo?' sino ‘Cuáles son los compromisos políticos e intelectuales que motivan nuestra investigación, y cómo estos dan forma no sólo a nuestra producción académica sino también a cómo elegimos denominarla?"' En estos términos, a Cohn (20l I, p. 583), al igual que ocurre con Wibben, le preocupa, en sus palabras, que "no puedo progresar en los problemas que más me interesa mejorar dentro de los confines de los estudios de seguridad" y por ello renuncia a no invertir excesiva energía en comprometerse de forma personal.

En el corazón de este debate se encuentra el sí,y el cómo, los EFS deben relacionarse con el subcampo de las RRII generalmente conocido como "Estudios de Seguridad" (que, de forma amplia pero no exclusiva, define la corriente principal estadounidense) y con las RRII en general. En mi opinión, tanto mi postura original, que estaba orientada a la disciplina, de manera que tenía la intención de ser instrumental y temporal, pero no cumplía con esas condiciones, como la posición de que los EFS no deberían involucrarse con la disciplina de manera más amplia, son ambas sobrelimitantes. Aunque estoy de acuerdo con Wibben en que los EFS no deben "aceptar los límites ontológicos, epistemológicos y metodológicos existentes en el campo" y deben 
entender el género como "transformacional de los estudios de guerra tradicionales," también considero que ignorar la estrechez y poder de las estructuras disciplinarias existentes no los hace desaparecer (Sjoberg, 2013, pp. 282-283). A fin de cuentas, pues, mi futuro trabajo en los EFS se involucrará con 'la disciplina', aunque siempre defendiendo cuidadosamente el terreno de la política y la metodología feministas y abordando de forma explícita los sacrificios que el compromiso puede suponer.

Sin embargo, tampoco creo que mis EFS tengan que ser los EFS de todos - de hecho, creo que los EFS serían significativamente pobres intelectualmente si se restringiesen al intento de ofrecer una narrativa de seguridad, una dirección de producción académica, y/o una sola manera de entender su lugar en la(s) disciplina(s) en las que se sitúan-. En su lugar, creo que debe haber una producción de los EFS que esté orientada a las RRII, una producción que esté orientada a los estudios de género, una producción que esté orientada a la política global, una producción que esté orientada a los márgenes de la política global, y una producción que sea internamente referencial. Lo que esta variedad productiva compartiría/podría compartir/comparte es una orientación explícitamente feminista - un interés en llamar la atención y corregir la subordinación de género en la política global- Esta variedad proporciona, donde la uniformidad no podría, traducibilidad intelectual y política a una serie de audiencias diferentes para una serie de propósitos diferentes. Si los EFS van a crear un espacio disciplinario (en un lado de este debate) o lograr objetivos intelectualmente transformadores (en el otro lado), lo hacen más eficazmente flanqueados por una amplia variedad de enfoques que muestran su flexibilidad, su amplia aplicabilidad y su durabilidad.

\section{Estudios Feministas de Seguridad y Representación}

Trabajos tempranos en las RRII feministas enfatizaron un compromiso político de prestar atención a los márgenes de la política global, tanto en términos de localización de las mujeres como en cualquier sitio donde el desempoderamiento se encuentre (p. ej., Brown, 1988; Tickner, 1992; Peterson y Runyan, 1992). Mucho del trabajo en las RRII feministas, pues, es lo que los estudios de género podrían llamar interseccional: centrado en la intersección del género, la raza, la nacionalidad, y la clase en la política global (p. ej., Yuval-Davis, 1997; Moon, 1997; Chin, 1998). Este trabajo interseccional tiene un fuerte componente de feminismos poscoloniales/decoloniales (p. ej., Chowdhury y Nair, 2002;Agathangelou y Ling, 2004; Bueno-Hansen, 20 I 5; Picq, 20 I 8), observando las formas en las que las dinámicas en la política global han estado y siguen estando sexualizadas, en función del género, racializadas y colonialistas. Sin embargo, las problemáticas decoloniales no están completamente integradas en la producción académica de los EFS en algunas ocasiones, y algunas académicas feministas decoloniales (p. ej., Teaiwa y Slatter, 2013; D'Costa y Lee-Koo, 2013;Agathangelou, 2017; Medie y Kang, 2018) han expresado su inquietud con relación a que el lenguaje y las estructuras en desarrollo en los EFS contienen estructuras racializadas y clasistas.

Teaiwa y Slatter (20I3) sugieren que la propia construcción márgenes/centro, utilizada a menudo por las académicas en los EFS/RRII feministas, es una causa del problema más que una manera de llamar la atención hacia el mismo. Tal y como explican:"¿Las recientes articulaciones de los estudios feministas de seguridad (EFS) sugieren que las Islas del Pacífico son geopolíticamente marginales en el campo de estudio? ¿Cómo pueden las feministas reproducir todavía estas 
jerarquías de conocimiento y autoridad en pleno siglo $X X I$ ? ¿O es que quizás las jerarquías son endémicas a los Estudios de Seguridad?" (Teaiwa y Slatter, 20I3, p. 447). Observando los EFS, Teaiwa se pregunta "¿debemos lamentar la ausencia de un cuerpo coherente de EFS sobre las Islas del Pacífico o sentirnos afortunadas por haber evitado una visión tan sesgada de lo que vale la pena conocer?" (2013, p. 447).

Mientras Teaiwa y Slatter se centran en los problemas que esto produce de la comprensión del conocimiento, D'Costa y Lee-Koo (20I3, p. 45I) exploran de forma explícita las potenciales implicaciones de una noción occidentalocéntrica de los EFS para los objetivos de la investigación feminista en la política global'. Argumentan que:

Se ha señalado que, como los estudios de seguridad de manera más amplia, los estudios feministas de seguridad están teorizados en inglés y localizados primariamente en el Norte Global. Este foro nos ofrece un espacio para la autoreflexión y para preguntarnos a nosotras mismas si realmente hemos tenido éxito al representar y/o incluir las voces de mujeres que habían sido previamente invisibles a través del cristal de la seguridad. ¿Estamos hablando con nosotras mismas o realmente marcado una diferencia? ¿Cuáles son las decisiones éticas que tomamos como académicas feministas críticas para ser inclusivas? Si de hecho nos comprometemos con el lenguaje de la inclusión, iqué papel desempeñamos en la política de la voz y, en particular, en la política de citación?

Laura Shepherd (2013, p. 437) debate las lecciones aprendidas:"Yo plantearía, sin embargo, que sigamos atentas a la cuestión de quién llega a formar parte de la conversación, y plantearía cuáles objeciones son vistas como desafíos legítimos a las formas dominantes de conocimiento tanto dentro como fuera de los EFS.'

Prestar atención a estas cuestiones requiere no solo señalar que existe exclusividad en los EFS orientados a la disciplina, sino también reconocer una gran variedad de otras disparidades. Las lagunas de recursos y la estructura de las instituciones de asociación académicas sirven a la representación excesiva de algunas perspectivas y la representación insuficiente de otras (lo que corresponde al empoderamiento relativo de algunas perspectivas y al desempoderamiento relativo de otras). El poder disciplinario tiene efectos disciplinantes. Tal y como la disciplina de los EFS critica, perspectivas relativamente empoderadas dentro de los EFS tienen una tendencia consciente $o$ inconsciente a privilegiar ciertas formas de conocimiento (a menudo aquellas que reflejan los modelos disciplinarios de investigación de las ciencias sociales occidentales). Es importante interrogar, criticar y ampliar estas asunciones. Este no es solo el caso de la representación entre los académicos en los EFS, sino también de qué temas se estudian y cómo. Oleadas recientes de producción académica feminista y queer decolonial han demostrado que

El artículo original contenía una explicación bastante extensa de la sección Política y perspectivas críticas de género que abordaban estos artículos, que Jennifer Lobasz y yo (20II) coeditamos. Se ha eliminado de este borrador porque es repetitivo de otros trabajos publicados (por ejemplo, Sjoberg, 20I4) y tangencial hasta el punto de identificar problemas de / con diversidad sustantiva y representativa en/con FSS. 
alejarse de las perspectivas blancas y europeas trae diferentes interpretaciones de conceptos existentes y muestra la importancia de ideas previamente desatendidas (p. ej., Cottet y Picq, 20 I9; Rao, 2020).

Solía creer que la pregunta apropiada sería ‘cómo serían unos EFS representativos?', pero me he convencido de que esta es una pregunta inadecuada para abordar las exclusividades de los EFS por tres razones. Primero, como he argumentado antes, con Teiawa y Slatter (20I3), no creo que haya ni deba haber unos EFS que sean unidireccionales, unificados o incluso unificables. En cambio, creo que puede haber muchos enfoques y perspectivas diferentes sobre los EFS, desde muchos lugares diferentes del mundo y muchas situaciones diferentes en esos lugares diferentes. Esto hace que las preguntas sobre unos EFS representativos sean demasiado limitadas. En segundo lugar, sin embargo, me he dado cuenta cada vez más de la importancia de no confundir la pluralidad con la distribución del poder. Como tal, preguntar por la representación no es suficiente porque la representación no es suficiente. Es inapropiado ignorar las dinámicas de poder que hacen que una posición pluralista sea políticamente problemática. En cambio, el trabajo de los EFS realizado desde posiciones de privilegio de todo tipo necesita reconocer y reflexionar sobre ese privilegio en cada paso posible. La inclusividad no puede consistir simplemente en contar cuántas personas de dónde se ven y qué están "contribuyendo" a conversaciones iguales en teoría. Tiene que tratarse fundamentalmente de repensar las teorías, ideas, definiciones y metodologías en los EFS que son posibles gracias a su exclusividad en general y su blancura específicamente, $y$ a reconstruir unos EFS descolonizados. Si uno de los principios clave de los EFS es buscar tanto una definición más amplia de seguridad como una comprensión más profunda de las múltiples formas en las que el género y la seguridad son coconstitutivos, entonces ver las múltiples formas diferentes en las que los dos interactúan en diferentes contextos alrededor del mundo debe ser una parte crucial de esa investigación.

En tercer lugar, cuestionar cómo sería unos EFS representativos es una pregunta inadecuada porque no puedo saberlo. Puedo tener una idea de lo que falta, particularmente en términos de intersecciones de género, raza, clase, nacionalidades, sexualidades y religiones. Puedo ver las formas en las que los EFS se enriquece con material previamente excluido, ya sea sobre sexualidad (p. ej., Weber, 20I6), raza (p. ej., Howell, 20I8) o colonialidad (Kunz, 20I7). Pero un proyecto de "recuperación" para las exclusiones de los EFS es imposible (en el sentido de que no se puede construir el contrafactual de la inclusión) y no se puede aproximar en los experimentos mentales de una feminista occidental blanca. Pensar en cómo sería la inclusión no es un sustituto del trabajo real de descolonizar los EFS, y no debe confundirse como tal. Como muestran las discusiones recientes sobre el racismo en los conceptos y paisajes intelectuales incluso de trabajos críticos en Estudios de Seguridad (p. ej., Howell y Richter-Montpetit, 2019), es probable que múltiples capas de repensar y descolonizar sigan siendo rompecabezas difíciles para los EFS en los próximos años. Los proyectos de inclusión, traducción y viajes son importantes, pero no pueden salvar por sí solos estas importantes brechas.

\section{Estudios de Seguridad Feministas y Economía Política Feminista}

Una de las formas importantes en las que el género y la seguridad interactúan es mediadas por la 
economía política. A medida que la terminología y los focos de investigación de los EFS se hicieron más populares en el campo, también se desarrolló un campo de EPF, quizás buscando delimitar el territorio para un análisis económico continuo y en desarrollo. Durante los primeros años del uso popular del término EFS, tuve la sensación de que el trabajo de EFS estaba sobrerrepresentado en las presentaciones de conferencias y publicaciones de trabajos en las RRII feministas. La EPF como subcampo (quizás de la Economía Política Internacional y / o Global y de las RRII feministas) planteó el argumento de que la investigación de la economía política sobre el género y la política global es una parte esencial para comprender el género / RRII. Si bien, al igual que el término EFS, EPF como grupo de investigación inspiró una cantidad significativa de análisis intrincado, trabajo de campo cuidadoso, desarrollo teórico y recopilación de datos empíricos, también compartió con los EFS un sentido de especialización que no había caracterizado a las RRII feministas antes del uso explícito de la etiqueta EFS.

Particularmente, como mencioné anteriormente, el trabajo feminista temprano que abordó temas de seguridad en la política global reconoció y enfatizó las interrelaciones entre la economía política y la seguridad, especialmente en la medida en que entendía que la economía política desempeñaba un papel significativo en la seguridad en los márgenes de la política global, y las amenazas a la seguridad se manifiestan con frecuencia en efectos distributivos políticoeconómicos. La superposición que las primeras feministas en RRII vieron entre la economía política y la seguridad se había perdido durante más de una década a menudo en la investigación de EFS y EPF.

Para los EFS, aunque mi trabajo es tan susceptible a esta crítica como cualquier otro trabajo, si no más, encuentro cada vez más desafortunada esta bifurcación. La economía política siempre es relevante para las cuestiones de interés para los estudios de seguridad / EFS: las constelaciones políticoeconómicas son importantes para explicar cómo las experiencias de guerra y conflicto de las personas individuales; el desplazamiento relacionado con el conflicto; cómo los ejércitos son desplegados, armados y alimentados; quién perpetra la violencia contra quién; la dinámica social de unirse y luchar en el ejército; y a quién se siente amenazado por qué y cuándo en la arena política global. De hecho, como sostuve recientemente (Sjoberg, 2015, p. 4l0), muchas de las intersecciones más interesantes del feminismo y la seguridad son lugares donde no se debe ignorar la economía política, desde la naturaleza de género de las prácticas de logística militar hasta la comprensión de los impactos de género de la guerra en la salud. Separar los enfoques de "seguridad" y"economía política" de estos temas haría que cada uno de ellos estuviera incompleto.

Juanita Elias (20I5, p. 406) editó una Sección de Perspectivas Críticas de Politics and Gender para "observar la creciente división entre EFS y EPF con todos los participantes que buscan analizar cómo estas dos tradiciones de la producción académica feminista podrían reintegrarse y por qué esta reintegración es tan importante". Desde esa conversación inicial, creo que los EFS se han beneficiado sustancialmente del trabajo reciente que regresa a la integración del trabajo de seguridad y economía política que precedió al establecimiento del término EFS (p. ej., Martín de Almagro y Ryan, 2020; Lai, 2020; Tanyag, 2020; Basu, 2017). Aunque quiero y me gustaría mantener, una serie de desarrollos de la producción académica de los EFS, creo que revivir el sentido de interdependencia entre la economía política y la seguridad que existía en los primeros estudios feministas de RRII ha abierto un mundo completamente nuevo de trabajo teórico y explicativo para los EFS, y su potencial sólo ha comenzado a explorarse. 
La sensación de que los EFS se habían alejado del análisis de la economía política y, en ocasiones, lo habían dominado es importante, y creo que el futuro de los EFS deberían llevarlo aparejado, tanto sustancialmente, para una buena producción académica, como cuando se piensa en las políticas de representación sustantiva. En mi opinión, es importante que se realicen ciertos tipos de trabajo (por ejemplo, trabajo en seguridad 'dura', o dentro del subcampo estadounidense de estudios de seguridad, o similares), pero es igualmente importante que no estén sobrerrepresentados, y que no funcionen para silenciar la representación de otras líneas de investigación igualmente importantes (o quizás más importantes).Aquí, la investigación que podría ser silenciada o subrepresentada es el tipo de investigación que presta atención a la intersección de la economía política y la seguridad, la seguridad en los márgenes de la política global, las interpretaciones radicales de la necesidad de que la seguridad de las mujeres individuales sea empoderada en el discurso de la seguridad, y críticas a la securitización de lo que podría llamarse seguridad personal. Unos EFS que equilibren estas perspectivas, creo, es más probable que proporcionen información más útil a una variedad más amplia de personas, y que produzcan una investigación más interesante.

\section{Mirando hacia el futuro de los EFS}

Algunos pueden sugerir que el término 'Estudios Feministas de Seguridad' tiene demasiados antecedentes como para continuar usándolo como descriptor bien de un subcampo de las RRII feministas centrado en el estudio de la seguridad o de un subcampo de los Estudios de Seguridad en el seno del cual se realiza investigación. No me cuento entre estas personas. Aunque creo que es importante criticar, reimaginar, reconstruir, y descolonizar algunas de las exclusiones en el creciente campo de los EFS, creo que su autoidentificación como un campo ha abierto más espacio de los que ha cerrado. La proliferación de trabajos en EFS, y la propia existencia de estas contestaciones, demuestra tanto crecimiento como vitalidad.

A medida que cambian los EFS, también lo hace mi pensamiento. Como hice hace una década, pienso sobre el género como un importante elemento constitutivo, causal, y predictivo de los estudios de seguridad, y una pieza esencial de los proyectos basados en políticas. Pero considero la disputa y contestación ontológicas, epistemológicas y metodológicas dentro y en torno a los Estudios Feministas de Seguridad no como algo que debe ser tolerado con pluralismo o solucionado a través de la argumentación, sino como la sustancia del trabajo, que puede y debe desafiar a los Estudios de Seguridad, pero también puede y debe desafiar a las RRII, a las RRII feministas, a la economía política internacional, a los estudios de género, a la teoría queer, y a otras áreas de investigación relevantes para pensar sobre (y securitizar) los cuerpos de género como radical, sustantivamente y representativamente diverso, fuerte y autocrítico, y lo suficientemente amplio como para dar cuenta de la interdependencia entre la economía política y la seguridad. Espero que sea honesto sobre, y aborde directamente, sus racismos, sexismos, y heterosexismos (intencionales y no, representacionales y sustantivos).Además de aprender de estos debates, espero que los EFS continúen prestando particular atención a la teoría queer, la sexualidad y las personas LGBTQI en la política global; que mantenga su compromiso tanto con la teoría crítica como con el análisis de políticas, y que tienda la mano a una variedad aún mayor de comunidades de investigación de lo que lo hace en la actualidad. Al corazón de aquello en lo que los EFS se han convertido y se 
convertirán, sin embargo, radica, como siempre ha sido el caso, una comprensión feminista de lo que es la seguridad, y de cómo el género es constitutivo y se ve constituido por ella.

\section{Referencias}

Addams, Jane (1990) [1910]. Twenty Years at Hull-House. University of Illinois Press.

Agathangelou, Anna M. (20I7). "From Colonial to Feminist IR: Feminist IR Studies, the Wider FSS/GPE Research Agenda, and the Questions of Value,Valuation, Security, and Violence." Politics \& Gender 13(4): 739-746.

Agathangelou,Anna M. and L. H. M. Ling (2004)."Power, Borders, Security, and Wealth: Lessons of Violence and Desire from September I I.” International Studies Quarterly 48(3): 5 I 7-538.

Basu, Soumita (2017). "The UN Security Council and the Political Economy of WPS Resolutions". Politics \& Gender I3(4): $72 \mid-727$.

Brown, Sarah (1988).“Feminism, International Theory, and the International Relations of Gender Inequality.” Millennium: Journal of International Studies 17(3): 46I-475.

Bueno-Hansen, Pascha (2015). Feminist and Human Rights Struggles in Peru: Decolonizing Transitional Justice. University of Illinois Press.

Buzan, Barry, Ole Waever, and Jaap de Wilde (1998). Security:A New Framework for Analysis. Lynne Rienner.

Chin, Christine B. N (1998). In Service and Servitude: Foreign Female DomesticWorkers and the Malaysian Modernity Project. Columbia University Press.

Chowdhury, Geeta and Sheila Nair (2002). Power, Postcolonialism, and International Relations: Reading Race, Gender, and Class. Routledge.

Cohn, Carol (20I I).“'Feminist Security Studies':Toward a Reflexive Practice,” Politics \& Gender 7(4): 58 I-586.

Cottet, Caroline and Manuela Lavinas Picq (Eds.) (2019). Sexuality and Translation in World Politics. E-International Relations Publishing.

D’Costa, Bina and Katrina Lee-Koo (2013). "The Politics of Voice: Feminist Security Studies and the Asia-Pacific," International Studies Perspectives 14(4): 45 I-454.

Elshtain, Jean Bethke (1985). Women and War. University of Chicago Press.

Enloe, Cynthia (1989). Bananas, Beaches, and Bases: Making Feminist Sense of International Politics. University of California Press.

Enloe, Cynthia (2004). The Curious Feminist: Searching for Women in a New Age of Empire. University of California Press. Enloe, Cynthia (2010). Nimo's War, Emma's War: Making Feminist Sense of the Iraq War. University of California Press.

Elias, Juanita (Ed.) (20I5). “Feminist Security Studies and Feminist Political Economy: Crossing Divides and Rebuilding Bridges." Politics \& Gender I I (2): 406-438.

Elias, Juanita and Shirin Rai (20I5). "The Everyday Gendered Political Economy of Violence.” Politics \& Gender II(2): 424-429.

Haas, Peter M. (1990). Saving the Mediterranean: The Politics of International Environmental Cooperation. Columbia University Press.

Hansen, Lene (2000). "Gender, Nation, and Rape: Bosnia and the Construction of Security". International Feminist Journal of Politics 3(1): 55-75.

Harding, Sandra (1986). The Science Question in Feminism. Cornell University Press.

Howell,Alison (2018).“Forget 'Militarization': Race, Disability, and the 'Martial Politics' of the Police and the University.' International Feminist Journal of Politics 20(2): I I7-I36.

Hudson,Valerie M. (2009). "The Heart of the Matter:The Security of Women and the Security of States.” International Security 33(3): 7-45.

Husanovic, Jasmina and Patricia Owens (2000). "Emancipation: A 'Shrieking in Keeping'? An Email Conversation on Feminism, Emancipation, and Security between Jasmina Husanovic and Patricia Owens." International Feminist Journal of Politics 2(3): 424-434.

Keller, Evelyn Fox (1985). Reflections on Gender and Science. Yale University Press.

Keohane, Robert O. (1 984). After Hegemony: Cooperation and Discord in the World Political Economy. Princeton University Press.

Kunz, Rahel (2017). “Beyond the 'Helpless Nepali Woman' versus the 'Fierce Maoist Fighter': Challenging the Artificial Security/Economy Divide.” Politics \& Gender 1394): 733-739.

Lai, Daniela (2020). "Macroeconomic Interventions and the Politics of Postwar Justice," Politics \& Gender I6(3): el 3.

Lobasz, Jennifer and Laura Sjoberg, (Eds.) (20II). "The State of Feminist Security Studies," Critical Perspectives Section of Politics and Gender 7(4) (20I I): 573-604.

MacKinnon, Catharine (2006). Are Women Human? Harvard University Press.

Martin de Almagro, Maria and Caitlyn Ryan (2020)."Introduction: (Re)integrating Feminist Security Studies and Global Political Economy: Continuing the Conversation through Empirical Perspectives." Politics \& Gender 16(3): el 2.

Medie, Peace and Alice Kang (2018). "Power, Knowledge, and the Politics of Gender in the Global South." European Journal of Politics \& Gender I (I-2): 37-53. 
Mohanty, Chandra Talpede (1984).“Under Western Eyes: Feminist Scholarship and Colonial Discourses.” boundary 2 I2(3): 333-358.

Moon, Katherine (1997). Sex Among Allies. Columbia University Press.

Morgenthau, Hans (1948). Politics Among Nations:The Struggle for Power and Peace. McGraw-Hill.

Peterson,V. Spike (20I0). “A Long View of Globalization and Crisis.” Gloablizations 7(I-2): I 87-202.

Peterson, V. Spike (1992). “Transgressing Boundaries: Theories of Knowledge, Gender, and International Relations.” Millennium: Journal of International Studies 2 I (2): I83-206.

Peterson,V. Spike and Anne Sisson Runyan (1992). Global Gender Issues. Westview Press.

Pettman, Jan Jindy (1996). Worlding Women. Routledge.

Picq, Manuela (2018). Vernacular Sovereignties: Indigenous Women Challenging World Politics. University of Arizona Press.

Qiu Jin (1906). A Respectful Proclamation to China's 200 Million Women Comrades.

Rao, Rahul (2020). Out of Time:The Queer Politics of Postcoloniality. Oxford University Press.

Reardon, Betty (1985). Sexism and the War System. Teachers' College Press.

Shepherd, Laura (2013). “The State of Femiist Security Studies: Continuing the Conversation.” International Studies Perspectives I4(4): 436-439.

Shepherd, Laura (2009).“Gender,Violence, and Global Politics: Contemporary Debates in Feminist Security Studies.” Political Studies Review 7(2): 208-2I9.

Sjoberg, Laura (20I5). "From Unity to Divergence and Back Again: Security and Economy in Feminist International Relations." Politics \& Gender I I (2): 408-4I 3.

Sjoberg, Laura (20I4). "The Politics of Location and the Location of Politics:Thinking about Feminist Security Studies.' International Studies Perspectives I5(4): 566-569.

Sjoberg, Laura (2013). Gendering Global Conflict:Toward a Feminist Theory of War. Columbia University Press.

Sjoberg, Laura (2009). “Introduction to Security Studies: Feminist Contributions.” Security Studies I8(2): I84-2 I4.

Smith, Steve (2005). "The Contested Concept of Security," in Ken Booth, ed. Critical Security Studies in World Politics. Westview Press.

Sylvester, Christine (1994). Feminist International Relations in a Postmodern Era. Cambridge.

Tanyag, Maria (2020). “A Feminist Call to Be Radical: Linking Women's Health and Planetary Health.” Politics \& Gender 16(3): el6.

Teiawa,Theresia and Claire Slatter (2014)."Samting Nating: Pacific Waves at the Margins of Feminist Security Studies.” International Studies Perspectives 14(4): 447-450.

Tickner, J. Ann (1997). “You Just Don't Understand: Troubled Engagements between Feminists and IR Theorists." International Studies Quarterly 4I (4): 6I I-632.

Tickner, J. Ann (1992). Gender in International Relations: Feminist Perspectives on Achieving Global Security. Columbia University Press.

Tickner, J. Ann (1988). “Hans Morgenthau’s Principles of Political Realism: A Feminist Reformulation,” Millennium: Journal of International Studies 17(3): 429-440.

Truth, Sojourner (I85I). “Ain’t I A Woman?” Speech Delivered to the Women's Rights Convention, Old Stone Church, Akron, Ohio.

Waltz, Kenneth (1959). Man, the State, and War. Columbia University Press.

Weber, Cynthia. 2016. Queer International Relations: Sovereignty, Sexuality, and the Will to Knowledge. Oxford University Press.

Whitworth, Sandra (1997). Feminism and International Relations:Towards a Political Economy of Gender in Interstate and Non-Governmental Institutions. Macmillan.

Wibben, Annick (2010). Feminist Security Studies a Narrative Approach. Routledge.

Yuval-Davis, Nira (1997). Gender and Nation. Sage. 


\section{RELACIONES INTERNACIONALES}

Revista académica cuatrimestral de publicación electrónica Grupo de Estudios de Relaciones Internacionales (GERI)

Universidad Autónoma de Madrid, España

https://revistas.uam.es/relacionesinternacionales

ISSN 1699 - 3950

f facebook.com/RelacionesInternacionales

3. twitter.com/RRInternacional

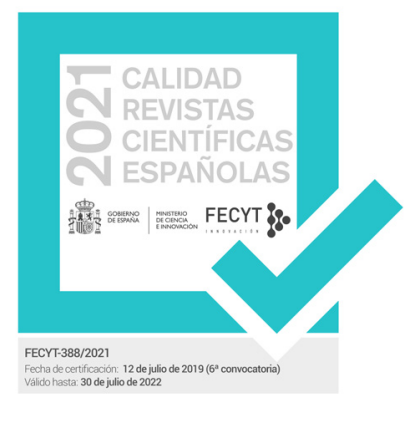

